\section{The feasibility of eradicating Aedes aegypti in the Americas ${ }^{1}$}

\section{INTRODUCTION}

In recent years considerable attention has been given to the serious threat posed by new, emerging, and re-emerging diseases. In addition to the appearance of several new pathogens which cause diseases of marked severity, old pathogens have reemerged and are having a considerable impact on the Americas. One of the latter is dengue virus, which is widely dispersed and has become an important problem in the Western Hemisphere.

These developments have rekindled an old and important public health debate: whether or not it is feasible to strive toward hemisphere-wide eradication of vector species-notably, the mosquito Aedes aegypti, which transmits both dengue and urban yellow fever. Eradication is defined as the total elimination of all stages of the mosquito (adults, pupae, larvae, and eggs) from every container on all premises in every infested area. It requires total coverage of all infested and potentially infested areas, both for eradication of the mosquito and for verification.

In response to a mandate given by the countries at the XXXVIII Meeting of the PAHO Directing Council in September 1995 (Resolution CD38.R12), PAHO convened a meeting of a technical task force to "study the feasibility, timeliness, and appropriateness of drawing up a hemispheric plan for the eradication of Aedes aegypti as an effective means of controlling dengue and urban yellow fever in the Americas." The task force met at the Fundação Oswaldo Cruz in Rio de Janeiro, Brazil, on 16-18 April 1996. Its findings were subsequently reported to the Governing Bodies of the Organization at their 1996 meetings.

\section{HISTORICAL BACKGROUND}

Activities directed against $A$. aegypti led to control of urban yellow fever throughout the Americas in the first half of the twentieth century. The objective of the early programs to combat yellow fever was to reduce the house infestation level of the vector to below 5\%-a level low enough that transmission was interrupted. Thus, even though these campaigns eliminated urban yellow fever, they did not eradicate $A$. aegypti.

The issue of whether eradication of $A$. aegypti was feasible has been debated since at least the 
1920s. In 1934, after having successfully eradicated the mosquito from several cities in the Northeast region, Brazil gave unofficial approval to a proposal for the eradication of $A$. aegypti within its borders, and in 1942 the Government of Brazil officially endorsed eradication. The I Meeting of the Directing Council of the Pan American Sanitary Organization (an earlier name of PAHO), held in Buenos Aires in October 1947, analyzed a joint report of Argentina, Bolivia, Brazil, and Paraguay on a plan for hemispheric eradication of $A$. aegypti proposed by Brazil. The Directing Council endorsed the proposal and resolved to entrust the Pan American Sanitary Bureau (PASB), the executive arm of the Organization, with the development of a program to accomplish this goal. Hemispheric eradication thereby became official policy for the express purpose of solving the problem of urban yellow fever; the resolution of the Directing Council made no reference of dengue.

The success of the hemispheric campaign organized by PASB was reflected in the fact that by 1962 , 18 continental countries and a number of Caribbean islands had achieved eradication. Several reasons for this success have been identified:

- National and external funding was adequate to provide well-trained personnel, necessary equipment, and insecticides.

- Country priorities were translated into political will.

- Total coverage of infested areas was achieved in time-limited programs.

- DDT was used for spraying in and around all breeding sites.

- Programs were streamlined and semiautonomous, and were separate from national health programs.

- Programs were centralized and vertically structured, with military-type organization and clear lines of command, strict supervision, and a high level of discipline.

Unfortunately, after 1962 only three additional countries or territories eliminated the vector. Even more serious, the countries that had achieved eradication began to be reinfested in the 1960s. The sources of reinfestation were countries that had not eradicated the mosquito (Cuba, the United States of America, Venezuela, and several Caribbean countries). Over time, in most of the countries that had achieved eradication the programs against A. aegypti lost political importance, and surveillance gradually declined so that small reinfestations could no longer be detected. In addition, once a reinfestation was discovered, the response to it was often slow because of the centralized structure of the program. Some of the other reasons for the setback were insufficient environmental sanitation in the rapidly growing urban centers; rapid expansion of international and domestic travel, which favored the passive dispersion of the mosquito, especially in the egg stage; development of resistance by A. aegypti to DDT and other organochlorine insecticides; high cost of materials and wages; insufficient community participation or support from the health sector for the establishment of intersectoral programs; and unwillingness on the part of some governments to join in simultaneous programs.

From the inception of the A. aegypti eradication effort in 1947, PAHO gave continuity and strong support to the organization and implementation of national programs. In this context, efforts to maintain the necessary political support were of the utmost importance. Every year between 1958 and 1972, with the exception of 1968, the Member States of PAHO adopted resolutions that reiterated their commitment to the eradication policy. In 1985 the XXXI Meeting of the Directing Council adopted a resolution (CD31.R26) calling for control or eradication of $A$. aegypti, which is interpreted as ending the policy of species eradication in the Region.

\section{PRESENT SITUATION}

With the exceptions of Bermuda, Canada, Chile, and Uruguay, all the countries of the Americas are infested with $A$. aegypti. This mosquito, which typically breeds in artificial containers in urban environments, has also been found in natural breeding sites (tree holes, leaf axils) in 19 countries and has been discovered up to 500 meters away from human habitation.

The progressively wider dissemination of the vector observed in the Americas since the 1970s, including urban centers located in enzootic and epizootic areas of jungle yellow fever, has created the possibility of the re-emergence of urban yellow fever in this hemisphere. A severe outbreak of jungle yellow fever, causing almost 500 cases and 192 deaths, affected Peru in 1995 near urban centers infested with $A$. aegypti. This outbreak illustrates that the urbanization of the disease is a real threat.

The risk is potentially greater because of the presence of another mosquito, Aedes albopictus, in six countries of the Americas: Brazil, Dominican Republic, El Salvador, Guatemala, Mexico, and the United States of America. Laboratory studies show that this species, which invaded the Americas from Asia in the mid-1980s, can transmit yellow fever virus. Because A. albopictus can breed in the domes- 
tic and forest environments, it could potentially serve as a bridge between the jungle and urban cycles of yellow fever. There is a real possibility that A. albopictus will infest enzootic areas of yellow fever in the near future.

Of immediate concern is the dengue situation. The number of cases of dengue and dengue hemorrhagic fever (DHF) in the Americas has risen dramatically in the past several years, to a total of over 274000 in 1995. Between 1968 and 1980 there were 60 cases of DHF reported from 5 countries in the Region, whereas between 1981 and 1985 there were 37030 cases reported from 24 countries. The countries have spent millions of dollars in attempts to control recent dengue epidemics (Table 1), and PAHO has provided assistance in the form of resource mobilization, control guidelines, support for a dengue laboratory network, and other activities.

\section{Prevention and control activities}

A program to control dengue and A. aegypti should be integrated in its implementation and must include the following components:

- Epidemiologic surveillance to detect on a systematic and ongoing basis the occurrence of cases of dengue and DHF. A well-developed system that includes both clinical and laboratory surveillance, as well as active case-finding, allows for early detection of an increase in dengue cases, making it possible to orient vector control measures in order to prevent an epidemic.

- Education of the medical community in the diagnosis and clinical treatment of DHF.

- Entomological surveillance to monitor levels of A. aegypti infestation in order to orient vector control activities and evaluate their quality.

- Integrated vector control, which is the logical combination of all the available methods of control (environmental management, chemical control, and biological control) in the most effective, economical, and safe manner.

A control program with these components needs to be sustained indefinitely in order to be successful.

\section{Current Aedes aegypti programs}

In preparation for the meeting of the task force, an international working group prepared a questionnaire to obtain information from all of the countries of the Americas on subjects dealing with the
TABLE 1. Spending on dengue control activities in the countries of the Americas, 1995.

\begin{tabular}{|c|c|}
\hline $\begin{array}{l}\text { Countries and territories, } \\
\text { by geographic area }\end{array}$ & $\begin{array}{l}\text { Expenditure } \\
\text { (US\$) }\end{array}$ \\
\hline \multicolumn{2}{|l|}{ North America } \\
\hline Mexico $^{a}$ & 3000000 \\
\hline \multicolumn{2}{|l|}{ Central America } \\
\hline Belize $^{b}$ & 90000 \\
\hline Costa Ricab & 1500000 \\
\hline El Salvadorb & 1000000 \\
\hline Guatemalab & 2100000 \\
\hline Honduras $^{b}$ & 1000000 \\
\hline Nicaragua $^{b}$ & 500000 \\
\hline Panama $^{b}$ & 1294000 \\
\hline \multicolumn{2}{|l|}{ Caribbean } \\
\hline Cuba $^{a}$ & 20884658 \\
\hline Dominica $^{\mathrm{a}}$ & 41000 \\
\hline Dominican Republic ${ }^{b}$ & 231000 \\
\hline Puerto Rico ${ }^{a}$ & 1159342 \\
\hline Montserrat ${ }^{\mathrm{a}}$ & 50000 \\
\hline Saint Vincent and the Grenadines ${ }^{\mathrm{a}}$ & 132080 \\
\hline \multicolumn{2}{|l|}{ South America } \\
\hline Argentina $^{\mathrm{C}}$ & 5950000 \\
\hline Bolivia $^{c}$ & 250000 \\
\hline Brazilc & 58591825 \\
\hline Chile $^{c}$ & 12000 \\
\hline Colombia $^{\mathrm{c}}$ & 3610490 \\
\hline Ecuador $^{\mathrm{a}}$ & 1034483 \\
\hline Paraguayc $^{c}$ & 185000 \\
\hline Peruc $^{c}$ & 1200000 \\
\hline Uruguay $^{c}$ & 10000 \\
\hline Total & 103825798 \\
\hline
\end{tabular}

feasibility, timeliness, and appropriateness of eradication campaigns. The data presented below are from the 28 countries that responded to the questionnaire. (Belize, Brazil, Guatemala, and Haiti are not included.) In each country, the questionnaires were filled out by the person responsible for the national program for combatting dengue and Aedes, together with the PAHO staff member working in vector-borne disease control in that country. The answers on the questionnaires did not always agree with information found in official government reports, but are presented here without modification.

Entomological aspects. Eight countries have A. aegypti infestations in areas where there is no access via roads or waterways. Twelve countries have infestations where access is difficult or dangerous because of social problems (e.g., armed conflicts, slums). Refusal to allow health workers into houses to check for infestations is low in most countries (under 3\%), but in some areas it is as high as $30 \%$. 
Nineteen countries have a surveillance system for Aedes albopictus, and in 16 countries larvae found in containers are identified with a bacteriological microscope.

Economic aspects. Per capita expenditures for Aedes control programs are highest in the Caribbean (average of US\$2.03 per capita, maximum of \$4.70), less in Central America and Mexico (average of $\$ 0.57$, maximum of \$1.94), and lowest in South America (average of $\$ 0.29$, maximum of $\$ 0.65$ ).

Operational aspects. Twenty-one of the countries responding to the questionnaire have an active program to combat $A$. aegypti: in 19 countries the objective is to control the vector, while 2 countries (Cuba and Trinidad and Tobago) are seeking to eradicate it. Six countries have a vertically structured program and nine countries have a decentralized program; the other six countries are in the process of decentralization.

Resistance to several insecticides used in Aedes control (temephos, malathion, lambda-cyhalothrine) has been reported in the Caribbean area.

Legal aspects. In 13 countries, there is legislation for the establishment of an Aedes program. Fifteen countries have laws to permit the entrance of field personnel into houses. Eight countries levy fines on people or businesses that have potential or actual breeding sites on their property. Four countries have laws prohibiting the importation of used tires, which can contain mosquito eggs and larvae and contribute to their dispersion. Six countries require the treatment of used tires at the point of origin. Disinsectation of aircraft arriving from infested areas is done in 14 countries. Notification of dengue cases is obligatory in 21 countries.

Political aspects. The fight against dengue is reported to be a high priority in the national health policy of 21 countries. Fifteen countries said that eradication of the vector is compatible with national health policies and plans, and 12 countries consider that an eradication program is feasible, timely, and appropriate for them. The countries are not in complete agreement about the most appropriate structure for the eradication program: 15 think it should be decentralized, four vertical, and two "other." In addition to the two countries that considered their programs to be for eradication (Cuba and Trinidad and Tobago), only one other country (Argentina) foresaw the conversion of its program to an eradication program, and only one country (Cuba) said that it had sufficient resources for eradication. Possible types of external financing mentioned were donations (seven countries) and a combination of donations and loans (eight countries).

\section{RECOMMENDATIONS OF THE TASK FORCE}

The task force reviewed the historical background of A. aegypti eradication as well as the current status of the countries' programs, as indicated by the survey and statements at recent subregional meetings on the subject of dengue control. It was the consensus of the task force that it is timely and appropriate to consider drafting a hemispheric plan for the eradication of $A$. aegypti, because this mosquito continues to spread to new areas and the incidence of dengue and dengue hemorrhagic fever continues to increase throughout the Americas. Noting that Brazil had drafted a national plan for eradication, it considered the time appropriate for other countries to consider joining in a coordinated hemispheric effort.

The members of the task force agreed that the following were the key challenges that had to be addressed in order to determine the feasibility of drafting a hemispheric plan for the eradication of A. aegypti:

Magnitude of the problem. A clear definition of the magnitude of the problem is needed from the outset. The geographical extension of the infested areas, the size of the urban population affected, the penetration of infestation into rural areas, and the numbers of containers per premises are all greater now than during the eradication campaigns of the 1950s and 1960s.

Eradication strategy. Eradication requires universal coverage of every municipality, every building and dwelling, and every container in infested areas. The original eradication campaigns that succeeded in eliminating $A$. aegypti from 21 countries were vertically structured and rigorously supervised. Most of the countries in the Americas consider that future eradication programs should be decentralized. However, A. aegypti has never been eradicated from any country with a decentralized program. The efficacy of any new eradication strategy needs to be demonstrated in pilot projects.

Cost. The cost of future eradication efforts will probably be much greater than that of previous campaigns and much greater than present control 
costs. Realistic estimates of costs required to achieve the goal and identification of national and international sources of funding are needed. It should be noted that programs in which environmental sanitation is a major component will bring health and quality of life benefits in addition to eradication or control of $A$. aegypti.

Political will. As governments and government officials change, health priorities change. In order for eradication to be maintained, there must be political commitment to uninterrupted surveillance against reinfestation, which must continue indefinitely.

International coordination. For hemispheric eradication to be completed, all of the countries of the Americas, as well as European authorities responsible for territories in the Americas, must agree to eradicate. Countries that do not launch A. aegypti eradication programs would become possible sources of reinfestation, as happened during the early campaigns. Also, A. aegypti is present in great numbers in Asia, which poses the risk of reinfestation from that Region.

The majority of the task force members ( 8 of 12 ) agreed that the above-listed key challenges have been met or could be met in the near future, and that it is feasible to draft an eradication plan. The four dissenting members thought that certain unmet key challenges remained major obstacles, namely, lack of a proven eradication strategy or model, difficulty in achieving universal coverage, and uncertainty regarding international commitment. The task force also recommended that PAHO review the list of key challenges and provide an update on their status to the Member States.

Finally, the task force recognized and congratulated the Government of Brazil for its bold initiative in proposing eradication of $A$. aegypti in order to eliminate dengue and DHF in that country. The plan is decentralized and includes a large environmental sanitation component. The Government of Brazil was encouraged to continue with its efforts, to document its progress, and to keep other countries informed.

\section{DELIBERATIONS BY THE GOVERNING BODIES OF PAHO}

The proposal of a hemispheric plan was taken up at the 118th Meeting of the Executive Committee of PAHO in June 1996. At that meeting, Brazil and Cuba described their countries' $A$. aegypti eradication plans and programs and requested that the Executive Committee recommend to the PAHO Directing Council that a regional plan for eradication be drafted. Several members of the Executive Committee expressed reservations about the feasibility of eradication and suggested that control of the mosquito might be a more realistic goal. Among the reasons they cited was the existence of many competing health priorities in the Region, which might preclude devoting the necessary resources to eradication. Nevertheless, the Committee recognized the increasing danger posed by dengue and was convinced of the need to intensify efforts to combat the vector and to develop a common strategy. It particularly noted the need for a broadbased, public health approach which combined environmental sanitation, public health education, and integrated vector control as important features of a hemispheric plan to combat the vector.

The topic also generated much discussion at the XXXIX Meeting of the Directing Council in September 1996. There was consensus that countries should move toward the goal of eradication, given the dangers posed by the current dengue and DHF situation and the potential for re-emergence of urban yellow fever. The Council therefore adopted a resolution (CD39.R11) urging the Member Governments (a) to collaborate in the definition of the general guidelines for a hemispheric plan to expand and intensify efforts to combat Aedes aegypt $i$ with a view to its eventual eradication in the Americas through persistent country-level efforts; (b) to prepare national plans to that same end; (c) to utilize the mass media to inform and educate the population and strengthen community participation in the integrated activities to combat the vector; (d) to promote epidemiological, entomological, and environmental surveillance; and (e) to strengthen national laboratories so that they can support the hemispheric plan. The Director was requested, as resources permitted, to form a task force of professionals from the member countries to develop the hemispheric plan; to provide support and advise in the preparation and execution of the countries' national plans; to help mobilize external resources to support the countries in the execution of the hemispheric plan based on national plans of action; and to promote and support technical cooperation among the countries in their development of national plans that comprise the hemispheric plan. 